\title{
Electro-optic steering of random laser emission in liquid crystals
}

\author{
Gaetano Assanto, ${ }^{* 1,2}$ Sreekanth Perumbilavil, ${ }^{2}$ Armando Piccardi, ${ }^{1}$ and Martti Kauranen ${ }^{2}$ \\ ${ }^{l}$ NooEL-Nonlinear Optics and OptoElectronics Lab, University "Roma Tre", IT-00146 Rome, Italy \\ ${ }^{2}$ Laboratory of Photonics, Tampere University of Technology, FI-33101 Tampere, Finland
}

Received September 27, 2018; accepted December 28, 2018; published December 31, 2018

\begin{abstract}
Using an external low-frequency electric field applied to dye-doped nematic liquid crystals, we demonstrate that random lasing obtained by optical pumping can be steered in an angular direction by routing an all-optical waveguide able to collect the emitted light. By varying the applied voltage from 0 to $2 \mathrm{~V}$, we reduce the walk-off and sweep the random laser guided beam over 7 degrees.
\end{abstract}

Random lasers (RL) are cavity-less light sources relying on recurrent photon scattering in active disordered media with population inversion [1-3]. Liquid crystals are among various material systems where random lasing has been reported upon optical pumping, using suitable dye dopants to provide spontaneous emission and, in turn, amplified spontaneous emission (ASE) and laser emission when the pump levels are high enough to overcome absorption losses [4-10]. A major drawback of such media is the poor quality of the emitted RL profile. However, since selffocusing and soliton generation is available in nematic liquid crystals (NLC) when launching beams of arbitrary wavelengths, we introduced earlier a configuration able to substantially solve those issues with RL. A spatial soliton excited by a beam at a nonresonant wavelength (not absorbed), in fact, can effectively guide and collect the ASE produced by intense pump pulses resonant with the dye-doped mixture when launched collinearly with the pump [11-13]. The reorientational nonlinear response in the near-infrared (NIR) at $1064 \mathrm{~nm}$ and the RL emission obtained by a green pump at $532 \mathrm{~nm}$ with $6 \mathrm{~ns}$ pulses at a $20 \mathrm{~Hz}$ rep-rate were proven to allow for efficient lasing around $580 \mathrm{~nm}$, with a smooth transverse profile and directional emission supported by the soliton waveguide.

It is well established that reorientational spatial solitons in nematic liquid crystals (nematicons) can be routed at will when modifying the NLC properties, e. g., by external voltages [14-19], external beams [20-21], additional nematicons [22-23], external magnetic fields [24] and so on [25-27]. In this Letter we describe the angular sweep of random laser emission by applying an external voltage which, acting on the optic axis (or molecular director $\mathbf{n}$ ) of the dye-doped NLC, modifies the path of a NIR nematicon and the guided RL light.
We co-launched orthogonally polarized NIR and green pump beams at the entrance of a planar glass cell $(3 \mathrm{~cm}$ width across $y, 0.1 \mathrm{~mm}$ thickness across $x$ and $2 \mathrm{~mm}$ length across $\mathrm{z}$ ) filled with an E7 mixture (positive uniaxial with $n_{\|}=1.71$ and $n_{\perp}=1.52$ the refractive index eigenvalues for electric fields parallel and orthogonal to the optic axis $\mathbf{n}$, respectively, at room temperature) doped with Pyrromethene (PM597) at $0.3 \mathrm{wt} \%$. The optic axis $\mathbf{n}$ was anchored at 45 degrees in the $y z$-plane, and both beams had wavevectors along the $z$-axis and were focused to a radius close to $3 \mu \mathrm{m}$, with the NIR - an extraordinary wave and the green - an ordinary wave.

The NIR formed a nematicon, propagating in the principal $y z$-plane with a walk-off angle $\delta$ of about 7 degrees. The emitted RL light, polarized as an extraordinary wave, was confined by the nematicon and guided to the output facet of the cell in the same direction [13].

Figure 1 is the artistic rendering of the experimental configuration. On the right-hand side, it shows the photograph of a typical RL output for pump pulses of energy $0.51 \mu \mathrm{J}$ in the presence of a $6 \mathrm{~mW}$ NIR nematicon.

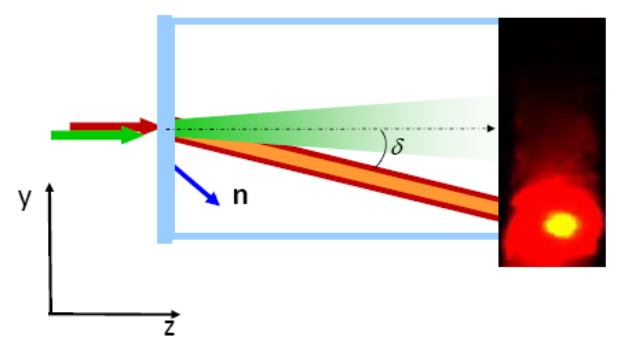

Fig. 1. Sketch of the experimental configuration. A green pulsed pump (green arrow) is launched along $\mathrm{z}$ as an ordinary wave, undergoing diffraction. An NIR continuous wave beam is co-launched as an extraordinary wave and generates a non-diffracting spatial soliton, propagating with walk-off $\delta$ and guiding the generated fluorescence, ASE and random laser emission around $580 \mathrm{~nm}$. The molecular director $\mathbf{n}$ is indicated by the blue arrow.

*E-mail: assanto@uniroma3.it 
The planar cell described above was also equipped with Indium Tin Oxide transparent electrodes, such that a voltage bias could be applied from an AC supply in order to reorient the optic axis electro-optically. This electric reorientation could pull the anisotropic NLC molecules out of the initial plane of anchoring $y z$, thereby rotating the principal plane versus voltage and the Poynting vector $\mathbf{S}$ of the soliton towards the wave-vector $\mathbf{k}_{\mathrm{NIR}}$ of the nematicon. Such voltage-driven reorientation, in analogy to what was reported in [14], produced an effective reduction of the observable walk-off in he observation plane yz, progressively realigning the spatial soliton to its wavevetor $\mathbf{k}_{\mathrm{NIR}} \| \mathbf{z}$. For applied (rms) voltages of about $2 \mathrm{~V}$, eventually, the walk-off vanished and the soliton waveguide channeled the RL emission along $\mathrm{z}$, realized a voltage-routable laser as sketched in Fig. 2.

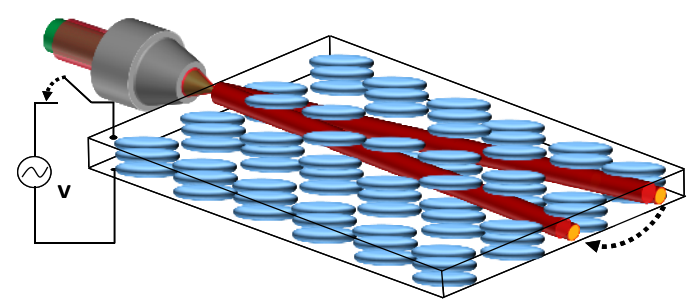

Fig. 2. Artistic rendering of the votage-steered random laser in nematic liquid crystals. As voltage $(\mathrm{V}=2 \mathrm{~V})$ is applied across the cell thickness (dashed arrows), the laser emission guided by the nematicon is redirected from $7^{\circ}$ to nearly $0^{\circ}$. The sketch shows also the microscope objective used to launch both pump and NIR beams in to the sample, along $z$.

Figure 3(a) shows typical output spots of the solitonguided random laser for $\mathrm{V}=0 \mathrm{~V}$ and for $\mathrm{V}=2 \mathrm{~V}$, respectively. The $\mathrm{RL}$ is effectively redirected as the applied bias acts directly on the laser medium, at variance with external means for steering laser emission in the presence of a standard or distributed cavity and outside it. Figure 3(b) graphs the measured angle of emission (in the observation $y z$-plane) versus the applied bias (squares with error bars), together with the calculated walk-off (solid blue line) based on the usual expression for uniaxial crystals:

$$
\delta=\frac{\left(n_{\|}^{2}-n_{\perp}^{2}\right) \sin 2 \Phi}{2\left(n_{\perp}^{2}+\left(n_{\|}^{2}-n_{\perp}^{2}\right) \cos ^{2} \Phi\right)}
$$

and projected on the observation $y z$-plane, i.e.,

$$
\delta_{y z}=\arctan \left(\frac{\tan \delta \cos \eta}{\sqrt{1+\sin ^{2} \eta \cot ^{2} \theta}}\right),
$$

with $\Phi=\arccos (\cos \eta \cos \theta)$ the angle between $\mathbf{k}_{\text {NIR }}$ and $\mathbf{n}$ upon electro-optic reorientation, $\theta$ - the initial orientation of $\mathbf{n}$ in $y z$, and $\eta$ - its elevation due to the bias across $x$. The angle $\eta$ can be calculated by integration of:

$$
\frac{d^{2} \eta}{d x^{2}}+\frac{\varepsilon_{0} \Delta \varepsilon_{\mathrm{LF}}}{2 K}\left(\frac{d V}{d x}\right)^{2} \sin (2 \eta)=0,
$$

with $\Delta \varepsilon_{\mathrm{LF}}$ the NLC dielectric anisotropy.

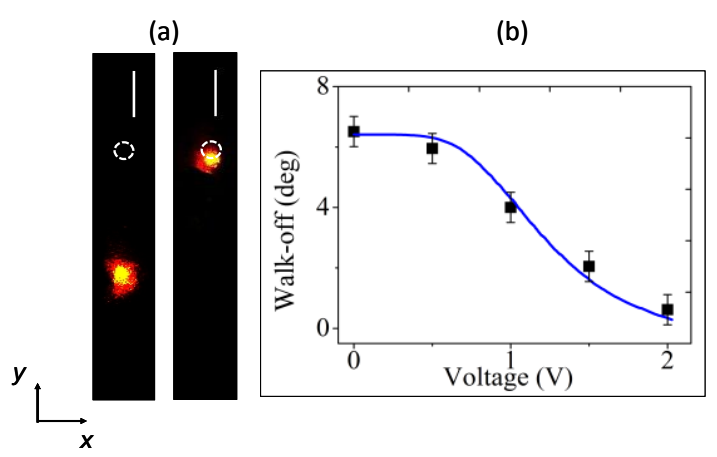

Fig. 3. (a) Photographs of random laser output (above threshold) in the presence of a pump-collinear $6 \mathrm{~mW}$ NIR nematicon, for (left) $\mathrm{V}=0 \mathrm{~V}$ and $\mathrm{V}=2 \mathrm{~V}$ (right). The white dashed circles indicate the output location of an ordinary-wave beam, i.e., along $\mathbf{k}_{\text {NIR. }}$ (b) Measured and calculated (solid line) output angle of the random laser beam versus applied voltage, corresponding to $\delta_{y z}$ from Eq. (2).

Finally, using a fiber-equipped spectrometer to acquire the RL emission spectra above the threshold $(>0.48 \mu \mathrm{J})$, we collected and averaged several spectra when in the presence of a $5 \mathrm{~mW}$ nematicon, at pump energies large enough to guarantee lasing even upon angular redirection, as a change in orientation $\theta$ is known to alter the reorientational nonlinear response of NLC [28]. Figure 4 summarized the results at pump energies of $1.1 \mu \mathrm{J}$ : the spectra show reduced efficiency as the beam is steered, presumably because of reduced soliton confinement as well as modified scattering within the interaction volume.

In conclusion, we have reported on the first laser where the direction of emitted light can be controlled by acting on the active medium itself with an external voltage. We have achieved this result by realizing a cavity-less random laser in PM597-doped nematic liquid crystals, whereby a near-infrared spatial soliton (a nematicon) could be launched collinearly with the pump and steered by modifying the birefringent walk-off. This effective alloptical waveguide was able to channel random laser emission and route it accordingly. 


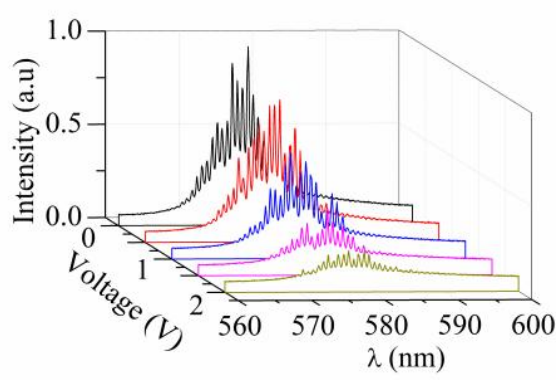

Fig. 4. Random laser emission spectra averaged over 200 realizations, versus applied voltage. Here the nematicon was injected with a $5 \mathrm{~mW}$ NIR beam and the pump pulse carried energies of $1.1 \mu \mathrm{J}$.

This work was supported by the Academy of Finland through the Finland Distinguished Professor grant no. 282858. The authors thank Sasha Buchnev and Pino Strangi for helpful suggestions and guidance.

\section{References}

[1] V.S. Letokhov, Sov. Phys. JETP 26 (4), 835 (1968).

[2] H. Cao, J.Y. Xu, D.Z. Zhang, S.-H. Chang, S.T. Ho, E.W. Seelig, X. Liu, R.P.H. Chang, Phys. Rev. Lett. 84 (24), 5584 (2000).

[3] D.S. Wiersma, Nature Phys. 4 (5), 359 (2008).

[4] D. Wiersma, S. Cavalieri, Nature 414, 708 (2001).

[5] G. Strangi, S. Ferjani, V. Barna, A. De Luca, N. Scaramuzza, C. Versace, C. Umeton, R. Bartolino, Opt. Expr. 14 (17), 7737 (2006).

[6] G. Strangi, S. Ferjani, V. Barna, A. De Luca, C. Versace, N. Scaramuzza, R. Bartolino, Proc. SPIE 6587, 65870P (2007).

[7] S. Ferjani, V. Barna, A. De Luca, C. Versace, G. Strangi, Opt Lett. 33(6), 557 (2008).

[8] S. Ferjani, L-V. Sorriso, V. Barna, A. De Luca, R. De Marco, G. Strangi, Phys. Rev. E 78 (1) 011707 (2008).

[9] H. Bian, F. Yao, H. Liu, F. Huang, Y. Pei, C. Hou, X. Sun, Liq Cryst. 41 (10), 1436 (2014).

[10] C.R. Lee, S.H. Lin, C.H. Guo, S.H. Chang, T.S. Mo, S.C. Chu, Opt. Expr. 18 (3), 2406 (2010).
[11] S. Perumbilavil, A. Piccardi, O. Buchnev, M. Kauranen, G. Strangi, G. Assanto, Appl. Phys. Lett. 109(16), 161105 (2016).

[12] S. Perumbilavil, A. Piccardi, O. Buchnev, M. Kauranen, G. Strangi, G. Assanto, Opt. Expr. 25 (5), 4672 (2017).

[13] S. Perumbilavil, A. Piccardi, R. Barboza, O. Buchnev, M. Kauranen, G. Strangi, G. Assanto, "Beaming random laser with soliton control," Nature Comm., in press (2018) DOI: 10.1038/s41467-018-06170-9.

[14] M. Peccianti, C. Conti, G. Assanto, A. De Luca, C. Umeton, Nature 432, 733 (2004).

[15] J. Beeckman, K. Neyts, M. Haeltermann, J. Opt. A - Pure Appl. Opt. 8 (2), 214 (2006)

[16] A. Piccardi, M. Peccianti, G. Assanto, A. Dyadyusha and M. Kaczmarek, Appl. Phys. Lett. 94, 091106 (2009).

[17] R. Barboza, A. Alberucci, G. Assanto, Opt. Lett. 36 (14), 2611 (2011).

[18] A. Piccardi, A. Alberucci, R. Barboza, O. Buchnev, M. Kaczmarek, G. Assanto, Appl. Phys. Lett. 100 (25) 251107 (2012).

[19] Y.V. Izdebskaya, Opt. Lett. 39(6), 1681 (2014).

[20] A. Pasquazi, A. Alberucci, M. Peccianti, G. Assanto, Appl. Phys. Lett. 87, 261104 (2005)

[21] S.V. Serak, N.V. Tabiryan, M. Peccianti, G. Assanto, IEEE Photon. Technol. Lett. 18(12), 1287 (2006).

[22] M. Peccianti, C. Conti, G. Assanto, A. De Luca, C. Umeton, Appl. Phys. Lett. 81(18), 3335 (2002)

[23] A. Fratalocchi, A. Piccardi, M. Peccianti, G. Assanto, Opt. Lett. 32(11), 1447 (2007).

[24] Y. Izdebskaya, V. Shvedov, G. Assanto, W. Krolikowski, Nat. Comm. 8, 14452 (2017).

[25] M. Peccianti, G. Assanto, Phys. Rep. 516, 147-208 (2012).

[26] Y. Izdebskaya, A. Desyatnikov, G. Assanto, Y. Kivshar, J. Opt. Soc. Am. B 30(6), 1432 (2013).

[27] U. Laudyn, M. Kwasny, F. Sala, M. Karpierz, N.F. Smyth, G. Assanto, Sci. Rep. 7, 12385 (2017).

[28] A. Alberucci, A. Piccardi, M. Peccianti, M. Kaczmarek, G. Assanto, Phys. Rev. A 82, 023806 (2010). 